\title{
Altered Cell to Cell Communication, Autophagy and Mitochondrial Dysfunction in a Model of Hepatocellular Carcinoma: Potential Protective Effects of Curcumin and Stem Cell Therapy
}

\author{
Ola M Tork*, Eman F Khaleel, Omnia M Abdelmaqsoud
}

\begin{abstract}
Background: Hepato-carcinogenesis is multifaceted in its molecular aspects. Among the interplaying agents are altered gap junctions, the proteasome/autophagy system, and mitochondria. The present experimental study was designed to outline the roles of these players and to investigate the tumor suppressive effects of curcumin with or without mesenchymal stem cells (MSCs) in hepatocellular carcinoma (HCC). Materials and Methods: Adult female albino rats were divided into normal controls and animals with $\mathrm{HCC}$ induced by diethyl-nitrosamine (DENA) and $\mathrm{CCl}_{4}$. Additional groups treated after $\mathrm{HCC}$ induction were: $\mathrm{Cur} / \mathrm{HCC}$ which received curcumin; MSCs/HCC which received MSCs; and Cur+MSCs/ HCC which received both curcumin and MSCs. For all groups there were histopathological examination and assessment of gene expression of connexin $43(\mathbf{C x 4 3})$, ubiquitin ligase-E3 (UCP-3), the autophagy marker LC3 and coenzyme-Q10 (Mito.Q10) mRNA by real time, reverse transcription-polymerase chain reaction, along with measurement of LC3II/LC3I ratio for estimation of autophagosome formation in the rat liver tissue. In addition, the serum levels of ALT, AST and alpha fetoprotein (AFP), together with the proinflammatory cytokines TNF $\alpha$ and IL-6, were determined in all groups. Results: Histopathological examination of liver tissue from animals which received DENA-CCl ${ }_{4}$ only revealed the presence of anaplastic carcinoma cells and macro-regenerative nodules. Administration of curcumin, MSCs; each alone or combined into rats after induction of HCC improved the histopathological picture. This was accompanied by significant reduction in $\alpha$-fetoprotein together with proinflammatory cytokines and significant decrease of various liver enzymes, in addition to upregulation of Cx43, UCP-3, LC3 and Mito.Q10 mRNA. Conclusions: Improvement of $\mathrm{Cx} 43$ expression, nonapoptotic cell death and mitochondrial function can repress tumor growth in HCC. Administration of curcumin and/or MSCs have tumor suppressive effects as they can target these mechanisms. However, further research is still needed to verify their effectiveness.
\end{abstract}

Keywords: Connexin - gap junction - liver neoplasm - alpha fetoprotein - ubiquitin

Asian Pac J Cancer Prev, 16 (18), 8271-8279

\section{Introduction}

Hepatocellular carcinoma (HCC) is a malignancy with extremely threatening prospects and a five-year survival rate reported below 9\% (Sherman, 2005). Orthotopic liver transplantation is the only truly curative therapy, although the risk of recurrence and development of metastases remains. Moreover, in case of unresectable tumor, treatment is even more limited, as HCC does not respond to chemotherapy, besides that the liver does not tolerate high doses of radiotherapy (Forner et al., 2012).

Chronic liver diseases are risk factors that may predispose to $\mathrm{HCC}$, as well as any factor that chronically and slowly damages the hepatocytes. This possibly happens via inducing mitosis and making the DNA of these cells more susceptible to genetic alterations (Cervello \& Montalto, 2006).
Gap junctions (GJs) provide a medium through which GJ intercellular communication (GJIC) is expressed; GJs are largely formed by connexin $(\mathrm{Cx})$ proteins. In the liver tissue, GJIC mainly involves three kinds of $\mathrm{Cx}-\mathrm{Cx} 26$, Cx32, and Cx43-whose distribution depends on cell type and location in the liver lobule (Kojima et al., 1997). It is widely accepted that GJIC plays important roles in growth control, cell proliferation, and differentiation. Furthermore, many authors believed that disorders of GJIC were involved in carcinogenesis (Mesnil et al., 2005).

Protein degradation in eukaryotic cells affects the cellcycle regulation proteins, tumor suppressors, oncogenes, transcription factors, in addition to proapoptotic and antiapoptotic proteins. There are 2 major intracellular pathways of protein degradation: the ubiquitin-proteasome system (UPS) and the autophagy-lysosome system (autophagy). The UPS-mediated proteolysis consists 
Ola M Tork et al

of 2 steps: ubiquitination and proteasome-mediated degradation (Sorokin et al., 2009).

The ubiquitination of a protein requires ATP, an activating enzyme, E1, a conjugating enzyme, E2, and an ubiquitin ligase, E3 (or UCP-3). The proteasome is a cylindrical cellular complex that recognizes proteins tagged by ubiquitin and contains enzymes which break down these proteins, while recycling ubiquitin. It has been pointed out that proteasome regulation (inhibition, activation, modulation) has the potential for contributing to the control of a number of diseases including cancer (Jankowska et al., 2013).

However, the role of autophagy, an alternative caspaseindependent cell death program (Rashmi etal.,2008), and its underlying molecular mechanism, is still controversial in cancer, especially in tumor progression. Autophagy involves lysosomal degradation of cytoplasmic and cellular organelles. During autophagy, an isolation membrane is initially formed. This membrane wraps some cytoplasmic contents and transforms into the autophagosome, which then fuses with the lysosome and degrades its contents (Mathew et al., 2007a). Autophagyrelated gene (ATG) products play essential roles in autophagy. Autophagosome formation is mediated by conjugation systems composed of ATG proteins, which culminate in the conjugation of ATG12 to ATG5 and conversion of a soluble form of microtubule-associated protein 1 light chain 3 (LC3-I) to another bound form: LC3-II (Mizushima, 2007).

There is increasing evidence that key oncogenes and tumor suppressors modulate mitochondrial dynamics through important signaling pathways. In addition, it was found that mitochondrial mass and function may vary between tumors and individuals, although the significance of these events for cancer are not fully understood (Boland et al., 2013). Co-enzyme Q10 (Co-Q10 or Mito- Q10) serves as an essential carrier for the electron transfer in the mitochondrial respiratory chain for the synthesis of ATP. Most cells are sensitive to Mito-Q10 deficiency. In fact, this deficiency has been implicated in several clinical disorders such as heart failure, hypertension, Parkinson's disease, and obesity. In this context, recent evidence suggests that Mito-Q10 supplementation may be useful for the treatment of obesity, oxidative stress, and the inflammatory process in metabolic syndrome (Machado et al., 2013).

In view of the limitations of the current treatment options available for HCC, novel approaches, especially chemoprevention, has been suggested as the key approach in lowering the occurrence and mortality associated with HCC (Kensler et al., 2003).

Phytochemicals have shown potential usefulness as therapeutic, as well as chemopreventive, agents for several chronic disorders such as metabolic, neoplastic and neurodegenerative diseases (Darvesh et al., 2010). Dietary polyphenols, such as catechins present in green tea, curcumin in turmeric, lycopene found in tomatoes, resveratrol in peanuts and many others, have shown considerable promise as both preventive and therapeutic agents in cancer affecting several organ systems such as breast, liver, lung, prostate and skin (Ullah \& Khan, 2008
; Khan\& Mukhtar, 2015).

The therapeutic effects of curcumin is due to its potent antioxidant, anti-inflammatory properties as well as its ability to modulate an array of signaling mechanisms (Sarkar et al., 2010).

In an animal model of hepatic injury, the researchers suggested that MSCs might become a more suitable source for Stem Cell based therapies than hepatic stem cells, because of their immunological properties and their highest potential for liver regeneration compared with other BM cell subpopulations (Cho et al., 2009).

To the best of our knowledge, little is known about the significance of alteration in Cx mRNA expression and its correlation with UPS, autophagy and mitochondrial dysfunction in hepatocellular carcinogenesis. We conducted this experimental study to investigate the correlation between these pathways in hepatocarcinogenesis and to investigate the effects of curcumin treatment, with or without MSCs, in a rat model of HCC.

\section{Materials and Methods}

\section{Experimental animals}

The present study was conducted on adult female rats inbred strain of matched age and weight (6 - 9 months \& $120-150 \mathrm{gm})$. The rats were inbred in the experimental animal unit, Faculty of Medicine-Cairo University. They were kept at room temperature and normal dark-light cycles, one per cage and were maintained according to the standard guidelines of Institutional Animal Care and Use Committee and after Institutional Review Board approval. Animals were fed a semi-purified diet that contained (gm/ $\mathrm{kg}$ ): 200 casein, 555 sucrose, 100 cellulose, 100 fat blends, 35 vitamin mix, and 35 mineral.

\section{Experimental protocol}

We randomly selected 6 rats as normal control group (Group 1), they were received $3 \mathrm{~mL}$ subcutaneous injection of corn oil once weekly for 6 weeks, then after 6 weeks of usual care, they received $1 \mathrm{~mL}$ of $0.7 \%$ carboxymethylcellulose (CMC) orally, three times/week for the next 8 weeks.

The remaining rats were used to establish $\mathrm{HCC}$ models. HCC was induced by a single intraperitoneal injection of diethyl-nitroseamine (DENA) (Sigma ) at a dose of $200 \mathrm{mg} / \mathrm{kg}$ body weight once followed by weekly subcutaneous injections of $\mathrm{CCl}_{4}$ (Sigma) $\mathrm{CCl}_{4} /$ corn oil $(1: 1, \mathrm{v} / \mathrm{v}) /$ was given at a dose of $3 \mathrm{~mL} / \mathrm{kg}$ body weight for 6 weeks (Dakshayani et al., 2005). These animals were left to continue on usual care for another 6 weeks. These HCC rat model were further subdivided randomly into 4 groups each include 6 rats.

Group (2): HCC group. They were sacrificed after withdrawal of blood samples.

Group (3): HCC+ curcumin; rats were given oral curcumin (Sigma) suspended in $0.7 \%$ CMC $(300 \mathrm{mg} / \mathrm{kg}$, three times/week) for 8 weeks after withdrawal of $\mathrm{CCl}_{4}$ (Khedr \& Khedr, 2014).

Group (4): HCC + MSCs; rats were injected intravenously once with MSCs in a dose of $3 \times 10^{6}$ cells /rat (Abdel Aziz et al., 2011), after withdrawal of $\mathrm{CCl}_{4}$. 
Cell to Cell Communication, Autophagy and Mitochondrial Dysfunction in HCC: Effects of Curcumin and Stem Cell Therapy

Group (5): HCC + curcumin /MSCs ; rats were injected with MSCs as in group (4) and given oral curcumin as in group (3) for 8 weeks after withdrawal of $\mathrm{CCl}_{4}$.

At the planned time blood samples were collected from all animals before sacrifice by cervical dislocation. Liver tissues were collected for histopathological examination by hematoxylin and eosin (HE) stained sections. Quantitative real time PCR gene expressions of Cx 43,LC3, Mito.Q10 \&,UCP-3 (ubiquitin ligase, E3) and assessments of serum AFP , IL-6 \&TNF $\alpha$ and levels of liver enzymes: ALT and AST were conducted for all groups.

Serum alanine aminotransferase (ALT)\& aspartate transaminase (AST) assay: ALT\&AST levels were determined using conventional laboratory methods

Detection of alpha fetoprotein , IL-6 \& TNF- $\alpha$ was done using Elisa kit supplied by (R\&D Quantikine USA) according to manufacturers' instruction.

Detection of Cx43, LC3, Mito.Q10 \&, UCP-3 gene expression by quantitative real time PCR (qRT-PCR):

\section{$R N A$ isolation and reverse transcription}

Total RNA was isolated from tissue homogenate using the high pure RNA isolation kit (Roche Diagnostics, Mannheim, Germany), then quantified on a NanoDrop ${ }^{\mathrm{TM}}$ 1000 Spectrophotometer (Thermo scientific Waltham, MA, USA). The OD260nm/280 nm ratio was among 1.9 to 2.0. RNA samples were further assessed by electrophoresis on $1.5 \%$ agarose gels, and then visualized under UV light after ethidium bromide staining. RNA samples were stored at $-80^{\circ} \mathrm{C}$ in aliquots until use. The cDNA was synthesized with $1 \mu \mathrm{g}$ total RNA and $1 \mu \mathrm{l}$ (T) 18 Oligo using Revert Aid First Strand cDNA Synthesis Kit (Fermentas Thermoscientific) according to manufacturer's instruction .

\section{Quantitative real time PCR ( $q P C R)$}

The qPCR was performed in an optical 96-well plate with an ABI PRISM 7500 fast sequence detection system (Applied Biosystems, Carlsbad, California) and universal cycling conditions min $95^{\circ} \mathrm{C}, 40$ cycles of $15 \mathrm{~s}$ at $95^{\circ} \mathrm{C}$ and $60 \mathrm{~s}$ at $\left.60^{\circ} \mathrm{C}\right)$. Each $10 \mu \mathrm{l}$ reaction contained $5 \mu \mathrm{l}$ SYBR Green Master Mix (Applied Biosystems), $0.3 \mu 1$ gene-specific forward and reverse primers $(10 \mu \mathrm{M}), 2.5$ $\mu 1 \mathrm{cDNA}$ and $1.9 \mu \mathrm{l}$ nuclease-free water. The sequences of PCR primer pairs used for each gene are shown in Table 1. The data were analyzed with the ABI Prism sequence detection system software and quantified using the v1.7 Sequence Detection Software from PE Biosystems (Foster City, CA). Relative expression of studied genes was calculated using the comparative threshold cycle method. All the values were normalized to the GAPDH genes (Livak \& Schmittgen, 2001).

\section{Table 1. Primer Sequences Used for Real Time PCR}

\section{Western blot analysis of LC3-I and LC3-II}

Part of the liver was homogenized with RIPA buffer containing $5 \mathrm{M} \mathrm{NaCl}, 1 \mathrm{mM}$ phenylmethylsulfonyl fluoride (PMSF) , 10\% deoxycholic acid (DOC), 10\% SDS, $1 \mathrm{M}$ Tris ( $\mathrm{pH}$ 8.6). The tissue lysate was centrifuged at $12000 \mathrm{rpm}$ for $20 \mathrm{~min}$ at $4^{\circ} \mathrm{C}$. The lysate was then collected and protein concentration was determined with a BCA protein assay kit (Thermo Fisher Scientific Inc., USA). An aliquot of $7.5 \mu \mathrm{g}$ protein of each sample were denatured and then each sample was loaded on $8 \%$ sodium dodecyl sulphate-polyacrylamide gel electrophoresis and transferred to a nitrocellulose membrane (Amersham Bioscience, Piscataway, NJ, USA) using a semidry transfer apparatus (Bio-Rad, Hercules, CA, USA). The membranes were incubated with $5 \%$ milk blocking buffer containing $10 \mathrm{mM}$ Tris- $\mathrm{HCl}$ (pH 7.4), $150 \mathrm{mM} \mathrm{NaCl}$, and Tris-buffered saline with $0.05 \%$ Tween-20 (TBST) at $4{ }^{\circ} \mathrm{C}$ overnight. The membranes were then washed with TBST and incubated with a 1:1,000 dilution of anti- LC3-II or anti LC3-I antibodies (Thermo Fisher Scientific Inc., USA) for overnight on a roller shaker at $4^{\circ} \mathrm{C}$. The filters were washed and subsequently probed with horseradish peroxidase-conjugated goat anti-mouse immunoglobulin (Amersham. Life Science Inc., USA). Chemiluminescence detection was performed with the Amersham detection kit according to the manufacturer's protocols and exposed to $\mathrm{X}$-ray film. The amount of studied protein was quantified by densitometric analysis of the autoradiograms using a scanning laser densitometer (Biomed Instrument Inc., USA). The results were expressed after normalization for $\beta$-actin protein expression.

\section{Preparation of bone marrow (BM)-derived mesenchymal stem cells from rats}

Bone marrow was harvested by flushing the tibiae and femurs of 6-week-old male white albino rats with Dulbecco's modified Eagle's medium (DMEM, GIBCO/BRL) supplemented with $10 \%$ fetal bovine serum (GIBCO/BRL). Nucleated cells were isolated with a density gradient [Ficoll/Paque (Pharmacia)] and resuspended in complete culture medium supplemented with $1 \%$ penicillin-streptomycin (GIBCO/BRL). Cells were incubated at $37^{\circ} \mathrm{C}$ in $5 \%$ humidified $\mathrm{CO}_{2}$ for $12-$ 14 days as primary culture or upon formation of large colonies. When large colonies developed (80-90\% confluence), cultures were washed twice with phosphate buffer saline (PBS) and the cells were trypsinized with $0.25 \%$ trypsin in $1 \mathrm{mM}$ EDTA (GIBCO/BRL) for $5 \mathrm{~min}$ at $37^{\circ} \mathrm{C}$. After centrifugation, the cells were resuspended in serum supplemented medium and incubated in 50 $\mathrm{cm}^{2}$ culture flasks (Falcon). The resulting cultures were referred to as first-passage cultures (Abdel Aziz et al.,

\begin{tabular}{ll}
\hline Primer & Sequence \\
\hline CX-43 & Forward primer: 5'-GCGTGAGGAAAGTACCAAAC-3' Reverse primer: 5'-CCCGTTGGAACTCAAGAAGG-3' \\
UCP-3 & Forward: 5'- CCTGCCTCTCTCGGAAACAA-3' Reverse: 5'-TGTAGGCTGCCCAATGAACA-3' \\
Mito Q10 & Forward: 5'-GGGGAGGAAGATGTCAAAGC-3' Reverse: 5'-GCCTGAGACCCAATTGAAGG-3' \\
LC3 & Forward: 5'-CCTGCTGCTGGCCGTAGT-3' Reverse: 5'-CGCTGTACGAGGAACACCCCAGCT-3' \\
GAPDH & Forward: 5'- ATGGAAATCCCATCACCATCTT -3' Reverse: 5'- CGCCCCACTTGATTTTGG -3' \\
\hline
\end{tabular}

GAPDH= Glyceraldehyde 3 phosphate dehydrogenase 
Ola M Tork et al

2007). Cells was identified as being MSCs by their morphology, adherence, and their power to differentiate into osteocytes and chondrocytes. Surface marker for MSCs characterization like CD34, CD29\&CD105 was detected by flow cytometry analysis.

\section{Labeling of MSCs with PKH26}

MSCs were harvested during the 4th passage and were labeled with PKH26, which is a red fluorochrome. It has excitation $(551 \mathrm{~nm})$ and emission $(567 \mathrm{~nm})$ characteristics compatible with rhodamine or phycoerythrin detection systems. Labeled cells retain both biological and proliferating activity, and are ideal for in vitro cell labeling, in vitro proliferation studies and long in vivo cell tracking. In the current work, MSCs were labeled with PKH26from Sigma Company (Saint Louis, Missouri USA). Cells were centrifuged and washed twice in serum free medium. Cells were pelleted and suspended in dye solution. Cells were injected intravenously into rat tail vein. After one month, liver tissue was examined with a fluorescence microscope to detect and trace the cells.

\section{Analysis of liver pathology}

Liver samples were collected into PBS and fixed in $40 \mathrm{~g} / \mathrm{L}$ paraformaldehyde in PBS at $4^{\circ} \mathrm{C}$. Serial 5- $\mu \mathrm{m}$ sections of the right lobes of the livers were stained with hematoxylin and eosin (HE) and were examined histopathologically.

\section{Data treatment and statistics}

The data was coded and entered using the statistical package SPSS version 15 . The data was summarized using descriptive statistics: mean \pm standard deviation for all normally distributed variables or median and (interquartile range) for non parametric variables. Statistical differences between groups were tested using ANOVA (analysis of variance) for quantitative normally distributed variables. When a significant $\mathrm{F}$ was obtained, multiple comparison post tests were used to determine which groups were significantly different. Nonparametric Mann Whitney test was used for quantitative variables, which aren't normally distributed. Nonparametric correlations were done to test for linear relations between variables. P-values less than or equal to 0.05 were considered statistically significant.

\section{Results}

\section{MSCs characterization\& homing}

MSCs were identified by surface marker CD34 (ve), CD29 (+ve ) and CD105 (+ve) detected by flow cytometry (Figure 1A, B and C) respectively. MSCs labeled with PKH26 fluorescent dye were detected in the liver confirming that these cells homed into the liver tissue (Figure 2).

The Results of Liver Injury and Cancer Markers in Treated or not Treated Rats with induced HCC were summarized in Table 2

Levels of serum ALT and AST were significantly increased in the $\mathrm{DEN} / \mathrm{CCl}_{4}$-induced tumor control group compared to the normal control group ( $\mathrm{p}<0.001)$.
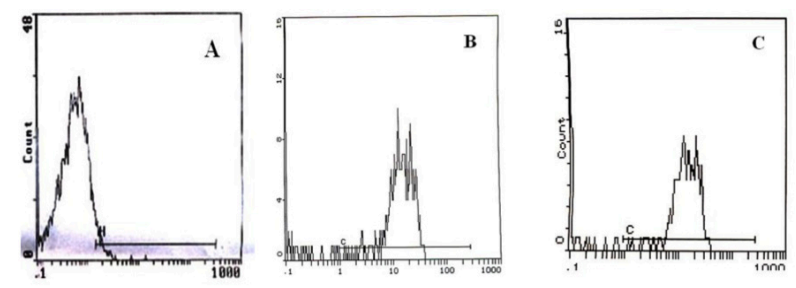

Figure 1. Characteristics of BM-MSCs. Cells were stained with the CD34, CD29 \& CD105 antibody and analyzed by flow cytometry. The expression levels of CD34-ve (A), CD29 +ve (B) CD105+ve (C), of BM-MSCs are presented as a histogram. The percentage of expression of the indicated markers was defined in the figure

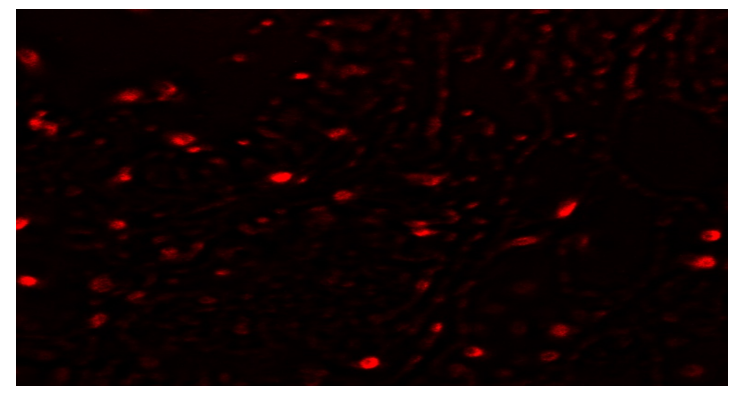

Figure 2. Detection of MSCs Labelled with PKH26 Fluorescent dye in Liver Tissue

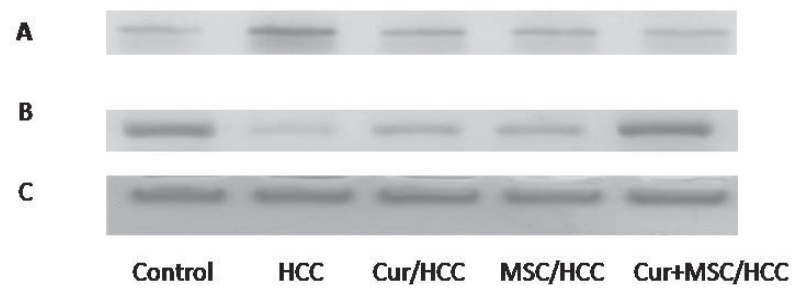

Figure 3. Western Blot Analysis of Protein Expression of LC3 -II (A),LC3-I (B)\& Beta Actin (C) in Different Studied groups

However, curcumin and MSCs treatment produced comparable effects in reducing markedly these liver enzymes but to a level still higher than that of the control $(\mathrm{P}<0.001)$. Interestingly, the combined treatment produced significant improvement in ALT\& AST when compared with curcumin treatment alone $(\mathrm{p}<0.01 \& \mathrm{p}<0.001$ respectively), or MSCs treatment alone $(\mathrm{p}<0.05)$ for AST only. This indicated that there was a synergism between curcumin and MSCs therapy which could markedly improve the induced liver injury.

As regards the tumor marker, serum AFP, it showed marked increase in the HCC group compared to the normal control group . The levels of AFP were significantly reduced in all treated groups ( $\mathrm{p}<0.001$ for all) compared with HCC group. It's level in curcumin and MSCs treated groups alone and combined showed no statistical significance compared with the normal control group.

The Results of Serum Inflammatory Markers TNF $\alpha$ \& IL-6 were summarized in Table 2

There was significant elevation $(\mathrm{P}<0.001)$ of $\mathrm{TNF} \alpha$ together with IL-6 $(\mathrm{P}<0.001)$ in the HCC rats compared to the control animals.

As regards the effect of treatment on these inflammatory cytokines, there was significant comparable reduction in 
Cell to Cell Communication, Autophagy and Mitochondrial Dysfunction in HCC: Effects of Curcumin and Stem Cell Therapy Table 2. The Level of Serum ALT (U/l), AST(U/l), AFP; Alpha Feto-protein (ng/ml) TNF $\alpha$ (pg/ml) and IL-6 (pg/ $\mathrm{ml})$, in the Studied Groups in Mean $\pm \mathrm{SD}(\mathrm{n}=6$ in each group)

\begin{tabular}{|c|c|c|c|c|c|}
\hline Groups/Parameters & Control & $\mathrm{HCC}$ & Cur/HCC & $\mathrm{MSCs} / \mathrm{HCC}$ & $\mathrm{Cur}+\mathrm{MSCs} / \mathrm{HCC}$ \\
\hline ALT & $18.13 \pm 2.05$ & $79.90 \pm 6.18 *$ & $40.27 \pm 4.22 * \#$ & $35.27 \pm 3.63 * \#$ & $29.0 \pm 3.36^{* \# \$}$ \\
\hline AST & $14.77 \pm 3.06$ & $68.18 \pm 4.82 *$ & $40.58 \pm 2.73 * \#$ & $33.90 \pm 3.91 * \#$ & $25.27 \pm 5.20 * \# \$ @$ \\
\hline AFP & $0.41 \pm 0.14$ & $1.87 \pm 0.45^{*}$ & $0.81 \pm 0.18 \#$ & $0.65 \pm 0.19 \#$ & $0.46 \pm 0.12 \#$ \\
\hline $\mathrm{TNF} \alpha$ & $31.92 \pm 3.74$ & $112.82 \pm 9.91 *$ & $72.78 \pm 4.97 * \#$ & $63.02 \pm 6.25^{* \#}$ & $49.37 \pm 5.41 * \# \$ @$ \\
\hline IL-6 & $31.22 \pm 3.88$ & $113.82 \pm 4.51 *$ & $76.52 \pm 4.25 * \#$ & $64.80 \pm 11.78^{* \#}$ & 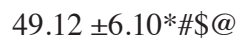 \\
\hline
\end{tabular}

*Significant compared to control at P-value $\leq 0.05$; \# Significant compared to HCC group at P-value $\leq 0.05 ; \$$ Significant compared to Cur/HCC group at $\mathrm{P}$-value $\leq 0.05$; @ Significant compared to MSCs/HCC group at $\mathrm{P}$-value $\leq 0.05$

Table 3. The Effect of Curcumin, MSCs Treatment Alone or Combined on LC3II/LC3I Ratio in Median and (interquartile range) and the Hepatic relative Expression of LC3 in (Mean \pm SD) and Connexin43, UCP-3, Mit. Q10 in Median and (interquartile range) in rat model of Hepatocellular Carcinoma (HCC). (n=6 in each group

\begin{tabular}{lccccc}
\hline Groups/Parameters & Control & HCC & Cur/HCC & MSCs/HCC & Cur+ MSCs/HCC \\
\hline LC3-II / LC3-I ratio $1.02(.97-1.09)$ & $38.15^{*}(30.88-45.38)$ & $10.55^{* \#(5.25-17.30)}$ & $5.58^{*} \#(5.49-16.58)$ & $2.14 * \# \$ @(2.08-2.48)$ \\
LC3 & $1.21 \pm 0.29$ & $0.24 \pm 0.08^{*}$ & $0.69 \pm 0.14 * \#$ & $0.78 \pm 0.16^{*} \#$ & $0.86 \pm 0.16^{*} \#$ \\
Cx43 & $1.25(0.99-1.50)$ & $0.15^{*}(0.13-0.39)$ & $0.48^{* \#(0.44-0.71)}$ & $0.66^{* \#(0.56-0.79)}$ & $0.92 * \# \$(0.64-0.95)$ \\
UCP-3 & $10.35(9.71-12.13)$ & $2.00^{*}(1.68-2.82)$ & $5.46 * \#(4.88-6.15)$ & $6.50^{* \#(5.23-7.03)}$ & $7.65 * \#(6.67-8.38)$ \\
Mito.Q10 & $1.13(1.00-1.43)$ & $0.23^{*}(0.18-0.51)$ & $0.69^{* \#(0.54-0.78)}$ & $0.76^{*}(0.48-0.82)$ & $0.83 * \#(0.70-0.87)$ \\
\hline
\end{tabular}

*Significant compared to control at P-value $\leq 0.05$; \# Significant compared to HCC group at P-value $\leq 0.05$; $\$$ Significant compared to Cur/HCC group at $\mathrm{P}$-value $\leq 0.05$; @ Significant compared to MSCs/HCC group at $\mathrm{P}$-value $\leq 0.05$
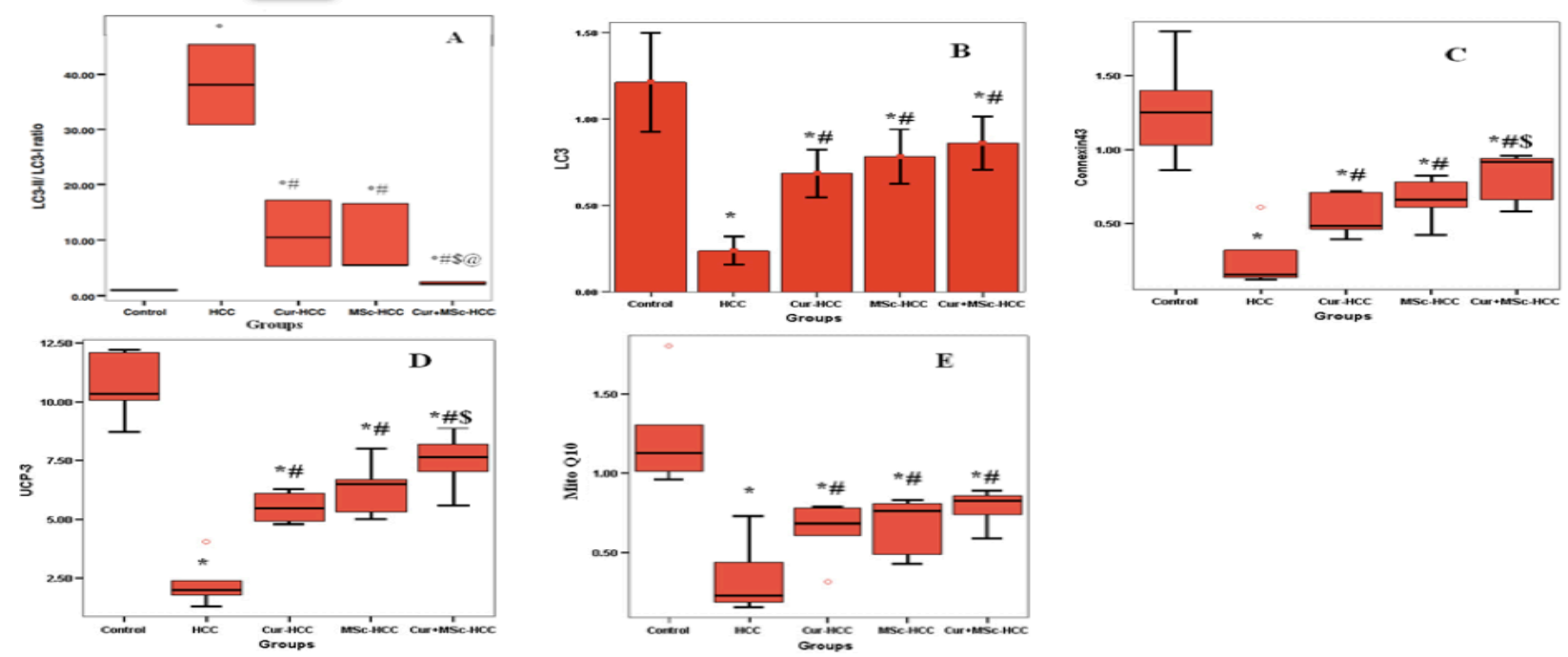

Figure 4. (A) The Effect of Curcumin, MSCs Treatments; Each Alone or Combined on LC3II/LC3I Ratio in Median and (Interquartile range) and (B) the Hepatic Relative Expression of LC3 in (Mean \pm SD) and (C) Connexin43, (D) UCP-3, (E)Mito.Q10 in Median and (interquartile range) in Rat Model of Hepatocellular Carcinoma. ( $\mathrm{n}=6$ in each group). *Significant Compared to Control at P-value $\leq \mathbf{0 . 0 5}$. \# Significant Compared to HCC group at P-value $\leq \mathbf{0 . 0 5}$. \$ Significant Compared to Cur/HCC Group at P-value $\leq 0.05$; @ Significant Compared to MSCs/HCC Group at P-value $\leq \mathbf{0 . 0 5}$

their levels $(\mathrm{P}<0.001)$, either under curcumin or MSCs therapy alone, compared with the HCC rats. However, there was further significant reduction under combined treatment when compared with curcumin treated $(\mathrm{P}<0.001)$ or MSCs treated rats alone $(\mathrm{P}<0.05)$. This again indicates the presence of synergistic effects between curcumin and MSCs therapy in reducing the production of inflammatory cytokines from tumor cells.

The results of Western blot analysis of LC3-II and LC3-I and their ratios were summarized in Figure 3, 4A and in Table 3

The liver of HCC rats showed a statistically significant rise $(\mathrm{P}<0.01)$ of LC3-II / LC3-I ratio . It was significantly decreased in both curcumin or MSCs treated rats compared to HCC, but they were still significantly higher than the control animals $(\mathrm{P}<0.01)$. While the combined treatment generally produced significant differences from either of the treatment approaches alone, but still higher than that of the control group.

The changes in the level of $\mathrm{mRNA}$ relative expression of Cx 43, UCP-3, LC3 and Mito.Q10 in studied groups were summarized in Table 3 and Figures 4 (B, C, D\& E):

The liver of HCC rats exhibited notably statistical significant decrease $(\mathrm{P}<0.01)$ of connexin43, UCP-3 and Mito.Q10 gene expression . They were significantly 


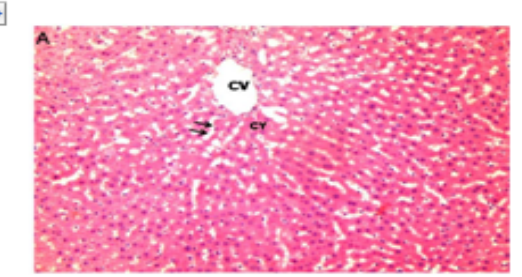

A

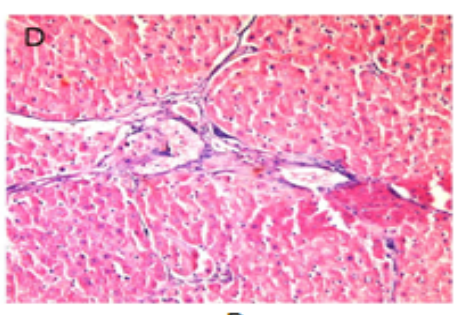

D

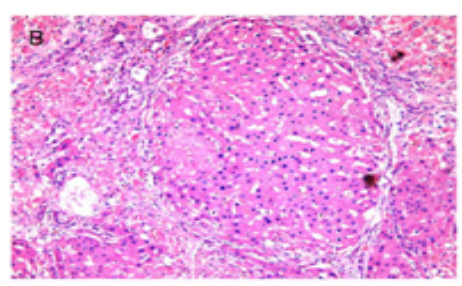

B

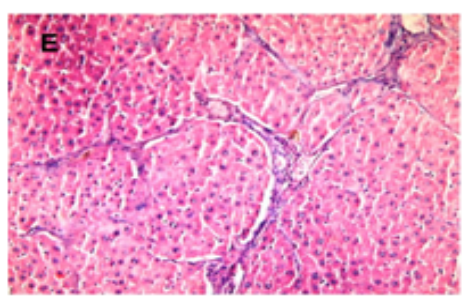

E

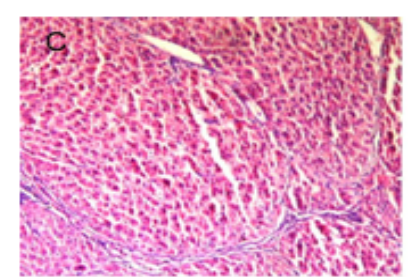

c

Figure 5. H\&E-stained Liver Tissue Biopsies from Rats of Experimental Groups (magnification factor $\times 200$ ). (A) normal control group: shows the architecture of a hepatic lobule of control rats. The central vein (CV) lies at the centre of the lobule surrounded by the hepatocytes with strongly eosinophilic granulated cytoplasm (CY), and distinct nuclei (N). Between the strands of hepatocytes the hepatic sinusoids are shown (black arrows). (B) HCC group: shows area of aberrant hepatocellular phenotype with variation in nuclear size, hyperchromatism, and irregular sinusoids, with prominent hyperbasophilic preneoplastic focal lesions and eosinophilic clear cell foci, the trabeculae of hepatocellular carcinoma consists of highly pleomorphic tumor cells and degenerated tumor cells. (C) Curcumin treated group $(300 \mathrm{mg} / \mathrm{kg}$ ) after induction of HCC; No nodularity \& liver appears normal with little necrosis. (D) MCSc treated group $\left(3 \times 10^{6}\right.$ cells /rat once I.V); No nodularity \& liver cells and lobules appear normal with ballooning degeneration, Normal portal tracts, No fibrosis, No inflammation, Area of cell drop out with stem cells. (E) Combined Curcumin and MCSc treated group: shows the hepatic lobule appear like the control one

elevated in both curcumin or MSCs treated rats $(\mathrm{P}<0.05)$ compared to HCC, but they were still significantly higher than the control animals $(\mathrm{P}<0.01)$. While the combined treatment generally produced insignificant differences from either of the treatment approaches alone , the levels of connexin43 and UCP-3 with combined treatment showed significant elevation compared to the curcumin treated rats alone $(\mathrm{P}<0.05)$.

In addition, there was significant decrease in the gene expression of the autophagic marker LC3 expression $(\mathrm{P}<0.001)$ in the liver of HCC rats compared with the control . However, whether curcumin or MSCs; each alone or combined treatments, all caused significant rise in LC3 gene expression compared to group II $(\mathrm{P}<0.001)$, yet still higher than the control level $(\mathrm{P}<0.001)$.

The results of the current study showed a positive correlation between the level of liver $\mathrm{Cx}-43$ expression and each of the following: UCP-3, LC3 and Mito.Q10 mRNA $(\mathrm{r}=0.805,0.746 \& 0.917$ respectively and $\mathrm{P}<0.001$ for all). On the other hand there was a significant inverse correlation between the gene expression of connexin 43 and liver enzymes ; ALT \& AST, inflammatory markers; TNF \& IL6 and AFP ( $\mathrm{r}=-0.824,-0.829,-0.848,-0.882$ $\&-.770$ respectively and $\mathrm{P}<0.001$ for all).

In the livers from the normal control group rats, hepatic lobules were structurally intact, hepatic cords were arranged in neat rows, and nuclei were clearly visible (Figure 5A). On the other hand, the livers from the tumor control group rats showed characteristics of hepatocellular carcinoma or neoplasm, including obvious nodular liver lesions, irregularly shaped or spindle-shaped cell changes, varying degrees of fatty degeneration, hyperchromatic and prominent nuclei and mitosis (Figure 5B).

Histopathological examination of the liver tissues showed marked improvement after treatment with curcumin and/or MSCs (Figures 5C- D\&E). The best results were found in the $\mathrm{HCC}$ rat group that received combined treatment of both MSCs and curcumin. The use of either curcumin or MSCs separately results in partial restoration of liver parenchyma with no evidence of the presence of malignant transformed hepatocytes.

\section{Discussion}

An aggressive cancer cell phenotype always results in recurrence or a poor prognosis. Thus, our study, which focuses on the combined role of GJIC ,UPS /autophagy and mitochondrial dysfunction in the pathogenesis of HCC, is extremely important. Numerous studies have revealed that a loss of control over genes contributing to these factors is closely associated with tumor progression, invasion and metastasis. As the onset of metastatic dissemination corresponds to the acquisition of specific traits by tumor cells affecting their migration and invasion (Liang et al., 1999 ; Aita et al., 1999; Mesnil et al., 2005; Jankowska et al., 2013; Boland et al., 2013).

In the present study we found that curcumin and MSCs administration caused significant improvement in the histopathologic pictures, liver enzymes, AFP together with Cx43, UCP-3, LC3 \& Mito.Q10 gene expression; the markers used for GJIC ,UPS /autophagy and mitochondrial dysfunction respectively; when compared to HCC group. The combined use of curcumin and MSCs gave more improvement than when each one was used alone in almost all the measured parameters. This might indicate a synergistic effect of both treatments.

The increase in serum ALT and AST in HCC group indicated that DENA/ $\mathrm{CCl}_{4}$ may induce acute liver injury. 
Cell to Cell Communication, Autophagy and Mitochondrial Dysfunction in HCC: Effects of Curcumin and Stem Cell Therapy

Therefore, during carcinogenesis, these enzymes could be used as biomarkers for HCC response to therapy according to Dakshayani et al (2005).

This may be due to the release of these enzymes from the cytoplasm into the blood rapidly after cellular damage, where variable forms of hepatocytes degeneration, apoptosis and necrosis were observed histopaholgically (Fu et al., 2008).

Serum AFP has been used as a clinical marker in the diagnosis and monitoring of HCC. In our work, there was a marked increase in the serum AFP level in tumor control group animals. This finding is consistent with the previous report that DENA-induced HCC in rats led to an increase in serum AFP (Liu et al., 2012).

The current study showed that there was significant increase in the serum levels of TNF $\alpha$ and IL-6 in the HCC group . The inflammatory cascade has been held as a key mediator of the pathogenesis of several chronic illnesses including neoplastic diseases and HCC (Packard\& Libby , 2008 ; Haybaeck etal, 2009).

Our finding that curcumin treatment suppressed inflammation by significantly reducing the levels of TNF- $\alpha$ and IL-6 was in accordance of Bassiouny et al.(2011). This could be attributed to the curcumin-mediated suppression of nuclear factor-KB (NF-KB), which is the master switch in the inflammatory cascade (Nishida et al., 2010). Curcumin can mediate its effect on TNF $\alpha$ expression by inhibiting p300/CREB-specific acetyl transferase, leading to repression of the acetylation of histone/nonhistone proteins and histone acetyl transferase-dependent chromatin transcription (Balasubramanyam et al., 2004). Moreover, curcumin could affect the expression of TNF $\alpha$ by affecting the methylation of a TNF $\alpha$ promoter (Reuter et al., 2011).

We showed that there was significant decrease in Cx43 in the HCC group. Cancerous cells generally display reduced GJIC activity (Yamasaki \& Naus, 1996). One of the mechanisms that underpin the loss of GJIC in carcinogenesis is mutations in Cx genes, which is considered an infrequent event. Instead, epigenetic modifications, such as DNA hypermethylation, may lead to silencing of connexin production (Shimizu et al., 2007).

In accordance with this hypothesis is the finding of Ionta and his colleagues, that artificial overexpression of Cx43 in cultured rat HCC cells was reported to decrease their proliferation rate (Ionta et al., 2009).

The relationship between autophagy and apoptosis within the tumor is complex. Initially, autophagy was considered to be an alternative pathway to cellular termination that was termed autophagic cell death. However, increasing evidence suggests that autophagy constitutes an adaptation to stress that avoids tumor death (and could even suppress apoptosis). On the other hand it was shown that simultaneous defects in autophagy and apoptosis may activate the DNA damage response in vitro, promote gene amplification and aneuploidy, and thus accelerate tumorigenesis (Mathew et al., 2007b). Thus, the loss of the pro-survival role of autophagy is likely to contribute to tumor progression by promoting genome damage and instability in an apoptosis-deficient background.
It was believed initially that UPS and autophagy were independent systems associated with different degradation mechanisms and with different physiologic characteristics. However, recent evidence suggests that they comprise 2 complementary and interlinked protein degradation pathways. Ubiquitinated proteins can be degraded by selective autophagy through p62 docking protein (Kirkin et al., 2009), and the autophagic protein LC 3 can be processed by the 20S proteasome (Gao et al., 2010). In addition, proteasome inhibitors induce misfolded proteins and protein aggregates to accumulate in the endoplasmic reticulum, leading to endoplasmic reticulum stress (Verfaillie et al., 2010), which, in turn, activates autophagy, resulting in the removal of potentially toxic, damaged proteins (Yorimitsu et al., 2006). In the study Yorimitsu et al.,(2006) two key autophagy genes (Beclin-1 and LC3) were up-regulated by proteasome inhibitors in a time-dependent manner in the HCC cell lines.

The previous studies might explain our results that the expression of autophagic gene LC3 and UCP-3 were extremely low in hepatic cells of HCC group. However, curcumin and MSCs treatment caused significant improvement in their expression compared with HCC rats and even more than when each one used alone, as they could act as direct proteasome inhibitors.

Then what may explain our results that showed rising LC3II/LC3I ratio in the HCC groups and their reduction under various treatments? It was mentioned that both an increase in autophagic flux and blockade of the downstream steps in autophagosomal maturation and lysosomal fusion may result in an increased number of autophagosomes (Takahashi et al., 2013). This blockade might explain our finding of elevated LC3II/LC3I ratio in HCC groups which was reduced markedly under curcumin and stem cell therapy specially when combined. This further supports our finding of inactivation of autophagy gene expression LC3in the HCC model.

In accordance with our findings is the work of Vara et al.,(2011) who provided evidence that in vivo studies revealed reduction in the growth of HCC subcutaneous xenografts under treatment due to induction of autophagy, an effect that was not evident when autophagy was genetically or pharmacologically inhibited in those tumors.

Further more, Ding etal., (2008) found that the relative Beclin 1 mRNA expression in a series of HCC patients with recurrent disease was significantly reduced compared with non-recurrent cases.

In our study, curcumin either alone or combined with MSCs was found to increase the transcription of the ubiquitin ligase E3. Actually, curcumin signaling in this system wasn't studied before. An explanation for the role of curcumin in this model is that it might directly inhibit the proteasome activity, then as a feedback increasing their transcription.

Little is known about the underlying molecular mechanisms that may link MSCs to the targeted inhibition of tumor cells. Qiao and coworkers (2008) reported that human mesenchymal stem cells (hMSCs) can home to tumor sites and inhibit the growth of tumor cells . Furthermore, other authors reported that hMSCs may 
inhibit the malignant phenotypes of the H7402 and HepG2 human liver cancer cell lines (Nakamizo et al., 2005).

A novel approach to deliver or stimulate the proliferation of healthy mitochondria involves the administration of stem cells. They were shown to target sites of mitochondrial dysfunction and to reduce a key hallmark feature associated with the disease (Nyamandi et al., 2014).

Recently Büeler,(2010) explored the interplay between the key molecules involved in mitochondrial fission and fusion and in apoptosis, as well as in mitophagy. He concluded that the coordinated induction of fission and autophagy is believed to facilitate the removal of damaged mitochondria through mitophagy .

We noticed an improvement of the expression of Mito-Q10 under curcumin and MSCs treatment. Abdin \& Hamouda (2008) found that Mito-Q10 may serve as an antioxidant by acting as a cofactor and activator of mitochondrial uncoupling proteins, leading to a reduction in free radical generation in vivo or via its activation of PPARs (Lee et al., 2012).

Conclusion: The essential premise for the potential use of curcumin and /or MSCs as a therapeutic agent in $\mathrm{HCC}$ rests on their potent anti-inflammatory properties as well as their ability to modulate a multitude of signaling mechanisms. It is clearly evident from our in vivo pre-clinical model that the synergistic effects between curcumin and MSCs therapy may hold great promise as a curative combination for HCC.

\section{References}

Abdel Aziz M, El Asmar M, Atta H, et al (2011). Efficacy of Mesenchymal Stem Cells in Suppression of Hepatocarcinorigenesis in Rats: Possible Role of Wnt Signaling. Journal of Experimental \& Clinical Cancer Research, 30, 49

Abdel Aziz M, Atta H, Mahfouz S, et al (2007). Therapeutic potential of bone marrow-derived mesenchymal stem cells on experimental liver fibrosis. Clin Biochem, 40, 893-9

Abdin A, Hamouda H (2008). Mechanism of the neuroprotective role of coenzyme Q10 with or without L-dopa in rotenoneinduced parkinsonism. Neuropharmacol, 55, 1340-6

Aita V, Liang X, Murty V, Pincus L, Yu W, Cayanis E, et al. (1999). Cloning and genomic organization of beclin 1, a candidate tumor suppressor gene on chromosome $17 \mathrm{q} 21$. Genomics, 59, 59-65

Balasubramanyam K, Varier R, Altaf M, et al (2004). Curcumin, a novel p300/CREB-binding protein-specific inhibitor of acetyl transferase, represses the acetylation of histone/ nonhistone proteins and histone acetyl transferase-dependent chromatin transcription. J Biol Chem, 279, 51163-71

Bassiouny A, Zaky A and Kandeel K (2011). Alteration of APendonuclease 1 expression in curcumin-treated fibrotic rats. Ann Hepatol, 10, 516-530

Boland M, Chourasia A and Macleod K (2013). Mitochondrial dysfunction in cancer. Front Oncol, 3, 1-28

Büeler H (2010). Mitochondrial dynamics, cell death and the pathogenesis of Parkinson's disease. Apoptosis, 15, 1336-53

Cervello M, Montalto G. (2006), Cyclooxygenases in hepatocellular carcinoma. World J Gastroenterol, 12, 5113-21

Cho K, Ju S, Cho S, et al (2009), Mesenchymal stem cells showed the highest potential for the regeneration of injured liver tissue compared with other subpopulations of the bone marrow. Cell Biology International, 33, 772-7

Dakshayani K, Subramanian P, Manivasagam T, et al (2005). Melatonin modulates the oxidant-antioxidant imbalance during N-nitrosodiethylamine induced hepatocarcinogenesis in rats. J Pharm Pharm Sci, 8, 316-21

Darvesh A, Carroll R, Bishayee A, et al (2010). Oxidative stress and Alzheimer's disease: dietary polyphenols as potential therapeutic agents. Exp Rev Neurother, 10, 729-745

Ding Z, Shi Y, Zhou J, et al (2008). Association of autophagy defect with a malignant phenotype and poor prognosis of hepatocellular carcinoma. Cancer Res, 68, 9167-75

Forner A, Llovet J M, Bruix J. (2012). Hepatocellular carcinoma. Lancet, 379, 1245-55

Fu Y, Zheng S, Lin J, et al (2008). Curcumin protects the rat liver from $\mathrm{ccl}_{4}$-caused injury and fibrogenesis by attenuating oxidative stress and suppressing inflammation. Mol Pharmacol, 73, 399-409

Gao Z, Gammoh N, Wong PM, et al (2010). Processing of autophagic protein LC3 by the $20 \mathrm{~S}$ proteasome. Autophagy, 6, 126-37

Haybaeck J, Zeller N, Wolf MJ, et al (2009). A lymphotoxindriven pathway to Hepatocellular carcinoma. Cancer Cell, 16, 295-308

Ionta M, Ferreira R, Pfister S, et al (2009). Exogenous Cx43 expression decrease cell proliferation rate in rat hepatocarcinoma cells independently of functional gap junction. Cancer Cell Int, 9, 22

Jankowska E, Stoj J, Karpowicz P, Osmulski P, et al (2013). The proteasome in health and disease. Curr Pharm Des, 19, 1010-28

Kensler T , Quain G , Chen J , et al (2003). Translational strategies for cancer prevention in liver. J Natl Cancer Inst, 3, 321-9

Khan N, and Mukhtar H (2015). Dietary agents for prevention and treatment of lung cancer. Cancer Letters, 395, 155-64

Khedr N , Khedr E (2014). Antioxidant and Anti-inflammatory effects of curcumin on $\mathrm{CCl}_{4}$ - induced liver fibrosis in rats. Am J Biomed Sci, 6, 191-200

Kirkin V, McEwan D, Novak I, et al (2009). A role for ubiquitin in selective autophagy. Mol Cell, 34, 259-69

Kojima T, Yamamoto M, Mochizuki C, et al (1997). Different changes in expression and function of connexin 26 and connexin 32 during DNA synthesis and redifferentiation in primary rat hepatocytes using a DMSO culture system. Hepatology, 26, 585-97

Lee S, Lee J and Kim J (2012). Coenzyme Q10 increases the fatty acid oxidation through AMPK-mediated PPAR $\alpha$ induction in 3 T3-L1 preadipocytes. Cell Signal, 24, 2329-36.

Liang X, Jackson S, Seaman M, et al (1999). Induction of autophagy and inhibition of tumorigenesis by beclin 1 . Nature, 402, 672-6

Liu J , Zhao H , Liu Y , et al (2012). Effect of two selenium sources on hepatocarcinogenesis and several angiogenic cytokines in diethylnitrosamine-induced hepatocarcinoma rats. J Trace Elem Med Biol, 26, 255-261

Livak K and Schmittgen T (2001). Analysis of relative gene expression data using real-time quantitative PCR and the 22DDCT. METHODS, 25, 402-8

Mathew R, Karantza-Wadsworth V and White E. (2007a). Role of autophagy in cancer. Nat Rev Cancer, 7, 961-7

Mathew R, Kongara S, Beaudoin B, et al (2007b). Autophagy suppresses tumor progression by limiting chromosomal instability. Genes Dev, 21, 1367-81

Mesnil M, Crespin S, Avanzo J, et al (2005). Defective gap junctional intercellular communication in the carcinogenic 
Cell to Cell Communication, Autophagy and Mitochondrial Dysfunction in HCC: Effects of Curcumin and Stem Cell Therapy process. Biochim Biophys Acta, 1719, 125-45

Mizushima N (2007). Autophagy: process and function. Genes Dev, 21, 2861-73

Nakamizo A, Marini F, Amano T, et al (2005). Human bone marrow derived mesenchymal stem cells in the treatment of gliomas. Cancer Res, 65, 3307-18

Nishida M, Nishiumi S, Mizushina Y, et al (2010). Monoacetylcurcumin strongly regulates inflammatory responses through inhibition of NF-kappaB activation. Int J Mol Med, 25, 761-7

Nyamandi VZ, Johnsen VL, Hughey CC, et al (2014). Enhanced stem cell engraftment and modulation of hepatic reactive oxygen species production in diet-induced obesity. Obesity (Silver Spring), 22, 721-9

Packard R., and Libby P (2008). Inflammation in atherosclerosis: from vascular biology to biomarker discovery and risk prediction. Clin Chem, 54, 24-38

Machado, C , Mendonça L, Venancio V, et al (2013). Coenzyme Q10 protects Pc12 cells from cisplatin-induced DNA damage and neurotoxicity. NeuroToxicology, 36, 10-16

Qiao L, Xu Z, Zhao Z, et al (2008). Suppression of tumorigenesis by human Mesenchymal Stem Cells in a hepatoma model. Cell Res, 18, 500-7

Rashmi R, Pillai SG, Vijayalingam S, et al (2008). BH3-only protein BIK induces caspase independent cell death with autophagic features in Bcl-2 null cells. Oncogene, 27, 1366-75

Reuter S, Gupta S, Park B, et al (2011). Epigenetic changes induced by curcumin and other natural compounds. Genes Nutr, 6, 93-108

Sarkar F , Li Y,Wang Z, et al (2010). Lesson learned from nature for the development of novel anti-cancer agents: implication of isoflavone, curcumin, and their synthetic analogs. Curr Pharm Des, 16, 1801-12

Sherman M (2005). Hepatocellular carcinoma: epidemiology, risk factors and screening. Semin Liver Dis, 25, 143-154

Shimizu K, Onishi M, Sugata E, et al (2007). Disturbance of DNA methylation patterns in the early phase of hepatocarcinogenesis induced by a choline-deficient L-amino acid-defi ned diet in rats. Cancer Sci, 98, 1318-22.

Sorokin A , Kim E and Ovchinnikov L (2009). Proteasome system of protein degradation and processing. Biochemistry (Mosc), 74, 1411-42

Takahashi W, Watanabe E, Fujimura L, et al (2013): Kinetics and protective role of autophagy in a mouse cecal ligation and puncture-induced sepsis. Critical Care, 17, 160

Ullah M and Khan M (2008). Food as Medicine: Potential therapeutic tendencies of plant derived polyphenolic compounds. Asian Pac J Cancer Prev, 9, 187-196

Vara D, Salazar M, Olea-Herrero N, et al (2011). Anti-tumoral action of cannabinoids on hepatocellular carcinoma: role of AMPK-dependent activation of autophagy. Cell Death and Differentiation, 18, 1099-1111

Verfaillie T, Salazar M, Velasco G, et al (2010). Linking ER stress to autophagy: potential implications for cancer therapy [serial online]. Int J Cell Biol, 2010, 930509

Yamasaki H and Naus C (1996). Role of connexin genes in growth control. Carcinogenesis, 17, 1199-213

Yorimitsu T, Nair U, Yang Z, et al (2006). Endoplasmic reticulum stress triggers autophagy. J Biol Chem, 281, 30299-304 